\title{
O direito à informação alimentar e nutricional em restaurantes: uma revisão
}

\section{The right to food and nutrition information in restaurants: a review}

\author{
Renata Carvalho de Oliveira' \\ Rossana Pacheco da Costa Proençal \\ Raquel Kuerten de Salles² \\ I Programa de Pós-graduação em Nutrição, \\ Universidade Federal de Santa Catarina (UFSC), \\ Núcleo de Pesquisa de Nutrição em Produção \\ de Refeições (NUPPRE), Florianópolis, Santa \\ Catarina, Brasil. \\ ${ }^{2}$ Departamento de Nutrição, Universidade \\ Federal de Santa Catarina (UFSC), Núcleo de \\ Pesquisa de Nutrição em Produção de Refeições \\ (NUPPRE), Florianópolis, Santa Catarina. Brasil. \\ Correspondência / Correspondence \\ Rossana Pacheco da Costa Proença \\ Departamento de Nutrição, Universidade Federal \\ de Santa Catarina (UFSC), Campus Trindade \\ 88.040-970. Florianópolis, SC, Brasil \\ E-mail: rossana@mboxl.ufsc.br
}

\section{Resumo}

A provisão de informação nutricional é uma das recomendações da Política Nacional de Alimentação e Nutrição (PNAN) e da Estratégia Global para promoção da Alimentação Saudável, Atividade Física e Saúde da Organização Mundial de Saúde (OMS), sendo já obrigatória para alimentos embalados no Brasil e em diversos países. Entretanto, o fornecimento de informação nutricional em restaurantes ainda não é obrigatório, nem uma prática comum, dificultando as escolhas alimentares dos consumidores de acordo com suas necessidades e seus hábitos quando se alimentam fora de casa. Este texto apresenta uma revisão sobre o direito do consumidor à informação alimentar e nutricional em restaurantes, considerando diferentes abordagens encontradas na literatura científica. Foram consultadas as bases de dados SciELO, Medline, Scopus e Portal da Capes, sites de órgãos oficiais nacionais e internacionais, assim como livros técnicos. Conclui-se que o fornecimento destas informações permite que as pessoas usufruam do seu direito a escolhas mais seguras e saudáveis, ao mesmo tempo em que atende às exigências da legislação e pode impulsionar investimentos da indústria de alimentos e de restaurantes na melhoria da qualidade nutricional dos alimentos e informações oferecidas.

Palavras-chave: Informação alimentar e nutricional. Rotulagem de alimentos. Legislação. Escolhas alimentares. Restaurante. 


\section{Abstract}

The provision of nutrition information is one of the issues recommended by the National Food and Nutrition Policy (PNAN) and Global Strategy on Diet, Physical Activity and Health from the World Health Organization (WHO), it is already mandatory for packaged food in Brazil and other countries. However, the provision of nutrition information in restaurants is not mandatory neither it is a common practice, making it harder for consumers to make food choices according to their needs and habits when eating away from home. This paper presents a review of the consumer's right to food and nutrition information in restaurants, considering different approaches found in literature. The databases SciELO, Medline, Scopus and Portal Capes were consulted, as well as, sites of national and international governmental institutions and technical books. It was concluded that providing this information allow people to enjoy their right to safer and healthier choices. At the same time it meets the requirements of the law and promotes increased investments by the food industry and restaurants, in improving the nutritional of food and information offered.

Key words: Food and nutrition information, Nutrition Labeling, Legislation, Food Choices, Restaurant.

\section{Introdução}

As informações alimentares e nutricionais disponibilizadas através de rotulagem constituem uma das formas de o consumidor se informar sobre a composição dos alimentos que está adquirindo. Pode propiciar, assim, a escolha alimentar de forma mais saudável e segura, considerando as necessidades nutricionais e os hábitos alimentares do indivíduo.

A informação nutricional é indicada na Política Nacional de Alimentação e Nutrição (PNAN) e na Estratégia Global para Promoção da Alimentação Saudável, Atividade Física e Saúde, da Organização
Mundial da Saúde (OMS) como uma das opções para fornecer à população informações precisas, padronizadas e compreensíveis sobre os alimentos (WHO, 2004a).

Sabe-se que a implementação da informação nutricional de alimentos embalados, na forma de rotulagem, é bastante recente no mundo, incluindo o Brasil (WHO, 2004b). Mas segundo a legislação brasileira, as informações nutricionais são obrigatórias apenas em rótulos de alimentos industrializados, excluindo os demais alimentos - por exemplo, os comercializados em restaurantes - de informarem a sua composição nutricional, tampouco seus ingredientes (BRASIL, 2003ํㅜㄹ). Neste contexto, 
apresenta-se uma revisão bibliográfica sobre o direito à informação alimentar e nutricional em restaurantes, considerando abordagens relacionadas à legislação de rotulagem de alimentos, à saúde coletiva e individual, bem como à questão cultural.

A pesquisa foi realizada por meio das bases de dados SciELO, Medline, Scopus e Portal da Capes, utilizando-se as palavraschave: informação nutricional em restaurante, rotulagem nutricional, informação ao consumidor, legislação sobre rotulagem nutricional e escolhas alimentares, nas línguas portuguesa, espanhola e inglesa. Foram considerados os artigos publicados no período de 1990 a 2011. Para complementar a discussão, foram consultados também sites de órgãos oficiais nacionais e internacionais, além de livros técnicos sobre o tema.

Informação alimentar e nutricional: abordagem relacionada à legislação sobre rotulagem de alimentos

No Brasil, a disponibilização de informação nutricional em rótulos de alimentos embalados tornou-se obrigatória a partir de 2001, para atender às exigências do PNAN, quanto ao aspecto nutricional, às questões relativas à composição, à qualidade e ao aproveitamento biológico dos alimentos (BRASIL, 2003b).

Além do Brasil e dos demais países do Mercosul (Mercado Comum do Sul), em outros países, a disponibilização destas informações também é obrigatória, como nos Estados Unidos da América, Canadá, Israel, Austrália,
Nova Zelândia e Malásia (WHO, 2004b). Todavia, a legislação vigente nesses países também torna obrigatória somente a presença de informação nutricional nos produtos embalados, não considerando a informação nutricional em restaurantes.

Nos Estados Unidos da América, o FDA (Food and Drugs Administration) sugere que os restaurantes norte-americanos incluam voluntariamente informações nutricionais nos locais de venda. Essa iniciativa objetiva promover práticas alimentares mais saudáveis, devido à associação entre a alimentação fora de casa, o aumento da ingestão de alimentos calóricos e a obesidade (FDA, 2004). Lá também já se encontram em vigor legislações municipais e estaduais que tornam obrigatórias a informação do valor calórico e/ou informação nutricional nos cardápios de restaurantes, como exemplo tem-se a cidade de Nova York e o estado da Califórnia (NYC, 2009; CALIFORNIA..., 2009). Em março de 2010, foi assinada a lei de reforma da Saúde (Health Care Reform Act), que incluiu a obrigatoriedade de informação nutricional em cadeias de restaurantes norte-americanos com mais de 20 unidades (USA, 2010).

Essas iniciativas buscam preencher uma lacuna visto que, segundo pesquisa realizada por Wootan; Osborn ${ }^{9}$, em 2006, apenas 54\% das 300 maiores cadeias de restaurantes daquele país ofereciam algum tipo de informação nutricional, sendo que a maioria das pequenas cadeias de restaurantes e pequenos estabelecimentos oferecia pouca ou nenhuma informação.

A disponibilização de informação nutricional nos rótulos de alimentos embalados, 
na União Europeia, é obrigatória somente para os alimentos que alegarem propriedades nutricionais. Mas as regras da Comissão Europeia sobre rotulagem se aplicam a alimentos embalados, bem como a todos aqueles fornecidos por restaurantes, hospitais, lanchonetes e similares. Além disso, a comissão está discutindo uma legislação para tornar a informação nutricional obrigatória (WHO, 2004b; CEC, 2008).

Estudo realizado com consumidores de restaurantes comerciais na Inglaterra apontou que estes são favoráveis à disponibilização de informações nutricionais nos cardápios. Porém, salientaram que a introdução destas informações deve estar associada a ações de educação, para que possam ser melhor compreendidas (ALEXANDER et al., 2010).

No Brasil, a RDC no 360 da ANVISA (Agência Nacional de Vigilância Sanitária), de 23 de dezembro de 2003, aplica-se à rotulagem de alimentos embalados. Esta legislação da ANVISA dispensa a disponibilização de informação alimentar e nutricional de alimentos preparados em restaurantes (BRASIL, 2003b). Contudo, em alguns locais do Brasil, identificaram-se legislações estaduais e municipais que tornam obrigatórias a disponibilização de informação nutricional em restaurantes ou a divulgação de determinados nutrientes.

Há uma lei, do ano de 2001, no Distrito Federal, que obriga os restaurantes self-service (autosserviço) e estabelecimentos similares a fixarem a quantidade média de calorias das porções dos alimentos (CÂMARA, 2009). Já em Santa Catarina, uma lei do ano de 2003 obriga as redes de refeições rápidas de opções restritas a informarem a seus clientes o valor calórico e informação nutricional contida nas suas refeições (ASSEMBLEIA, 2009). $\mathrm{E}$, mais recentemente, no mesmo estado, uma lei do ano de 2011 obriga a informar os ingredientes utilizados no preparo dos alimentos fornecidos por restaurantes, bares, lanchonetes e congêneres que comercializam e entregam em domicílio alimentos para prontoconsumo (ASSEMBLEIA, 2011).

No município do Rio de Janeiro/RJ, uma lei do ano de 2004 dispõe sobre a obrigatoriedade da divulgação da quantidade de calorias nos cardápios de bares, hotéis, restaurantes, fast foods (alimentação rápida) e similares (CÂMARA RJ, 2009). E, em Sorocaba/SP, desde 2005, está em vigor uma lei sobre a obrigatoriedade de restaurantes fast foods, bares, lanchonetes, traillers e estabelecimentos similares divulgarem informações e tabelas nutricionais sobre os alimentos que comercializam (CÂMARA SOROCABA, 2009).

Apesar da abrangência limitada dessas leis, que destacam em sua maioria a divulgação do valor calórico dos alimentos preparados em restaurantes e estabelecimentos afins, elas parecem refletir a crescente preocupação da população em relação à sua saúde e ao direito de informação sobre os alimentos consumidos fora de casa.

Estudos realizados por Benson (1995), Clay et al. (1995) e Fitzpatrick et al. (1997) mostraram que os consumidores podem influenciar positivamente os restaurantes a disponibilizarem informações nutricionais e a oferecerem um número maior de preparações 
mais saudáveis. Além disso, Jacobson (2004), em levantamento nacional realizado nos Estados Unidos, constatou que cerca de dois terços dos entrevistados gostariam de encontrar o valor calórico dos alimentos nos cardápios dos restaurantes em que costumam consumir as refeições. No mesmo país, Lando e LabinerWolfe (2007), em estudo com 68 consumidores divididos em grupos focais, observaram que os participantes reagiram favoravelmente à disponibilização de informações nutricionais em restaurantes quick-service (serviços rápidos).

$\mathrm{Na}$ Malásia, estudo realizado com consumidores de restaurantes revelou que estes consideram importante a disponibilização de informações nutricionais nos cardápios, sendo a informação de calorias, gorduras, proteínas e fibras as mais requisitadas (DIN et al., 2011). E Maestro e Salay (2008), em estudo realizado com 114 restaurantes na cidade de Campinas/ SP, constataram que apenas 25,4\% destes disponibilizavam informações nutricionais e/ou de saúde. A pesquisa constatou, ainda, que $46 \%$ dos estabelecimentos pesquisados têm interesse em disponibilizar informações nutricionais, com o objetivo de atrair novos clientes e fidelizar os já existentes.

Ressalta-se, contudo, que estas são iniciativas individuais dos proprietários de restaurantes e que, na maioria das vezes, não contratam serviços técnicos para padronizar as receitas, elaborar as fichas técnicas das preparações e auxiliá-los na elaboração das informações alimentares e nutricionais, requisitos considerados imprescindíveis para a correta disponibilização de informações alimentares e nutricionais em restaurantes.
Por conta disto, considera-se importante a existência de estímulos governamentais para incentivar os restaurantes a adotarem esta política, de forma a garantir informações corretas e seguras.

\section{Informação alimentar e nutricional:} abordagem relacionada à saúde da população

A Constituição Federal brasileira preconiza que "a saúde é um direito de todos e um dever do Estado, garantido mediante políticas sociais e econômicas que visem à redução do risco de doença e de outros agravos [...]” (BRASIL, 1988). Em fevereiro de 2010, foi aprovada a inclusão da alimentação como um dos direitos sociais previstos no artigo $6^{\circ}$ da referida Constituição (CÂMARA, 2010). E em 1999, foi homologada a Política Nacional de Alimentação e Nutrição (PNAN), que faz parte da Política Nacional de Saúde (PNS). A PNAN tem como objetivo contribuir com o conjunto de políticas públicas voltadas à concretização do direito universal à alimentação e nutrição adequadas e à garantia da segurança alimentar e nutricional da população brasileira (BRASIL, 2006a).

No ano de 2006, foi aprovada a Lei Orgânica de Segurança Alimentar e Nutricional brasileira (LOSAN), que trata do direito humano à alimentação adequada (BRASIL, 2006b). $\mathrm{O}$ texto desta lei faz menção à garantia da qualidade dos alimentos e seu aproveitamento e ao estímulo a práticas alimentares e estilos de vida saudáveis que respeitem a diversidade cultural da população brasileira (BRASIL, 2006b). Além disso, a busca da garantia do 
direito humano à alimentação, da segurança alimentar e nutricional e da consecução do objetivo de vida saudável estão contempladas na Estratégia Global para a Promoção da Alimentação Saudável, Atividade Física e Saúde, da OMS. A Estratégia Global tem como objetivo a prevenção e a promoção da saúde da população e o documento prevê parcerias para o seu desenvolvimento. Entre os parceiros citados, encontram-se os restaurantes, que podem ajudar a tornar as escolhas dos consumidores mais saudáveis, ao oferecer uma alimentação mais equilibrada nutricionalmente e fornecendo informações nutricionais das preparações oferecidas (WHO, 2004a).

Importante instrumento para o cumprimento das diretrizes da PNAN e da proposta da Estratégia Global, o Guia alimentar para a população brasileira contém as diretrizes alimentares oficiais do país para a prevenção e controle de doenças, estimulando a alimentação saudável na população (BRASIL, 2006a).

No contexto da promoção da alimentação saudável referido pela PNAN, pela Estratégia Global e pelo Guia alimentar brasileiro, a rotulagem nutricional pode ajudar o consumidor na garantia do seu direito à informação sobre os alimentos, fortalecendo-o na possibilidade de análise e de decisão para optar pela aquisição de alimentos mais saudáveis.

Alguns estudos demonstram que os consumidores estão mais propensos a consumir alimentos saudáveis quando são disponibilizadas informações nutricionais nos restaurantes (BURTON; CREYER, 2004; EDWARDS; MEISELMAN, 2005; STUBENITSKY et al., 2007). Burton et al (2006) perceberam que mesmo quando é disponibilizado apenas o valor calórico dos alimentos nos restaurantes, os consumidores procuram escolher aqueles alimentos com menos calorias, considerando que são mais saudáveis.

Hwang e Lorenzen (2008), em estudo realizado com consumidores de uma mercearia nos Estados Unidos da América, identificaram que os participantes apresentaram atitudes mais positivas em relação a alimentos com baixo teor de gordura, estando dispostos a pagar mais por esses itens, quando a informação nutricional era fornecida. Já Tandon et al. (2010) sugerem, em pesquisa realizada em um clínica pediátrica nos Estados Unidos, que a disponibilização de informações nutricionais nos cardápios de restaurantes pode levar à compra de refeições com menor valor calórico.

Corroborando, Nestle (2010), ao analisar a lei de reforma da Saúde nos Estados Unidos, afirma que a disponibilização de informações nutricionais, principalmente de calorias, pode ser um incentivo para que se reduza a venda de grandes porções pelas cadeias de fast food, pois o consumidor poderá comparar o valor calórico das diferentes preparações oferecidas pelos restaurantes. Desta forma, a disponibilização de rotulagem em restaurantes é importante para que a população possa garantir seu direito à informação sobre os alimentos, bem como ter acesso a informações que possam auxiliar seu entendimento sobre a importância da dieta na saúde. Assim, fortaleceria sua capacidade de análise e decisão para optar pela aquisição de alimentos saudáveis e poderia solicitar dos restaurantes a oferta de opções de preparações mais saudáveis. 
Informação alimentar e nutricional: abordagem relacionada à saúde do indivíduo

Existem diversas enfermidades relacionadas à alimentação que influenciam diretamente a escolha alimentar do indivíduo, pois necessidades especiais de seus portadores requerem restrição do consumo de determinados alimentos que, se ingeridos, podem desencadear problemas de saúde.

Dentre essas enfermidades, encontramse as alergias e intolerâncias alimentares, os distúrbios metabólicos e as doenças crônicas não-transmissíveis, como diabetes, hipertensão arterial e obesidade, e doenças relacionadas também ao consumo de gorduras, especialmente os ácidos graxos trans.

Para que as pessoas portadoras das enfermidades relacionadas possam fazer suas escolhas alimentares, é necessário que elas conheçam a composição dos alimentos e os ingredientes das preparações, tanto de alimentos embalados, quanto daqueles comercializados em restaurantes. Assim, na rotulagem de alimentos embalados, a informação sobre os ingredientes que podem estar associados a distúrbios e doenças relacionadas à alimentação e à nutrição é obrigatória na maioria dos países, incluindo o Brasil.

No Brasil, a Portaria SVS/MS no. 29/98 aprova o Regulamento Técnico referente a Alimentos para Fins Especiais, por exemplo, obrigando que os rótulos dos alimentos embalados informem a presença de fenilalanina e de mono e/ou dissacarídeos (BRASIL, 1998). Além desta, a Lei no $-10.674 / 2003$ obriga que os rótulos dos alimentos embalados informem a presença de glúten (BRASIL, 2003). E a Resolução RDC nํ..360/03 obriga a declaração do teor de gorduras saturadas e trans nas informações nutricionais ${ }^{4}$.

Neste contexto o papel da informação é fundamental, pois os consumidores não conseguem perceber diretamente o benefício ou malefício à saúde vindo dos alimentos, da mesma forma como podem perceber o sabor ou outras características sensoriais.

Em estudo realizado com familiares de pacientes com alergia ao leite de vaca, Binsfeld et al. (2009), constataram dificuldade na compreensão e identificação dos termos relacionados ao leite na rotulagem de alimentos industrializados. E segundo Thomas Jr.; Mills (2006), em pesquisa realizada com consumidores norte-americanos, a maior parte deles considera ser responsabilidade social do restaurante fornecer informações nutricionais detalhadas das preparações oferecidas, pois os alimentos podem beneficiar ou prejudicar a saúde.

Assim, para auxiliar as pessoas que seguem dietas com restrições alimentares, mais informações sobre os alimentos devem ser oferecidas, tanto nos alimentos embalados quanto nos restaurantes, para que as pessoas possam fazer suas escolhas alimentares de forma mais segura. Espera-se, no entanto, que tais informações, quando disponibilizadas, sejam confiáveis, pois somente assim auxiliarão efetivamente os consumidores. 
Informação alimentar e nutricional: abordagem cultural

O alimento, para ser capaz de manter a vida, não deve possuir apenas qualidades nutricionais, mas atender também a questões sensoriais e simbólicas. Assim, o alimento deve ser reconhecido e/ou aceito pelo indivíduo e por seu grupo social, pois muitas vezes comer se vincular mais a aspectos culturais e simbólicos relacionados aos alimentos do que às próprias necessidades fisiológicas do indivíduo (POULAIN, 2004; PROENÇA et al., 2005).

Pelas preferências alimentares, na escolha de um alimento em detrimento de outros é que se pode perceber que as pessoas não possuem um padrão alimentar uniforme, sendo influenciadas por questões culturais como costumes regionais, memória familiar, crenças, tabus, religiões, critérios de palatabilidade, entre outros (FISCHLER, 1988).

Essas questões são capazes até de impedir que alimentos existentes em abundância em determinada região sejam consumidos, podendo as pessoas enfrentar a fome ao invés de consumir alimentos não reconhecidos como comestíveis pela sua cultura. O que é alimento em uma cultura pode não o ser em outra, fato derivado não de seu valor nutritivo ou do perigo que oferece à saúde, mas de sua interpretação cultural (CARNEIRO, 2003).

O deslocamento das práticas alimentares dos indivíduos dos limites domésticos para o espaço público pode implicar alteração de certos hábitos alimentares previamente estruturados. Ao realizar a alimentação fora de casa, em especial em restaurantes de autosserviço, os consumidores se deparam tanto com dimensões relacionadas à ampla oferta de alimentos quanto com aquelas relacionadas às questões individuais. Essa situação os confronta com o dilema de ter que decidir onde, com quem e o que comer, diferentemente da situação doméstica, em que a escolha é geralmente determinada pelo responsável pela alimentação da família. Assim, o comer fora de casa apresenta às pessoas opções alimentares diferentes - às quais elas não estão acostumadas - e também a necessidade de confiar na qualidade do que lhes é oferecido nos restaurantes, com possibilidade de consequências para sua saúde (POULAIN, 2004; JOMORI et al., 2008).

Nesse sentido, pode ser um desafio para as pessoas comer fora de casa, tanto pela oferta de opções alimentares diferentes - às quais elas não estão acostumadas - quanto pela necessidade de confiar na composição e forma de preparo do que lhes é oferecido nos restaurantes.

Por isso, é importante que se disponibilize a informação sobre os tipos de alimentos oferecidos e a forma como são preparados nos estabelecimentos que comercializam alimentos. Tais informações permitem que as pessoas realizem suas escolhas alimentares respeitando sua cultura e suas preferências alimentares.

\section{Considerações finais}

Embora a disponibilização de informações alimentares e nutricionais atenda ao direito dos consumidores considerando as abordagens legais, de saúde e culturais, observa-se que 
as pessoas que se alimentam fora de casa em restaurantes não podem usufruir deste direito.

Mesmo que a disponibilização de informação nutricional seja obrigatória para alimentos embalados no Brasil e em diversos países, a provisão de informação nutricional em restaurantes ainda é facultativa e pouco frequente. Por este motivo, muitas vezes se torna difícil ter informação sobre os ingredientes e a composição nutricional das preparações oferecidas em cardápios ou bufês em restaurantes, pois há dificuldade visível para se identificar todos os ingredientes que compõem a preparação, não permitindo

\section{Referências}

ABREU, E.; SPINELLI, M.; ZANARDI, A. ALEXANDER, M.; O'GORMAN, K.; WOOD, K. Nutritional labelling in restaurants: Whose responsibility is it anyway? International Journal of Contemporary Hospitality Management, v.22, p.572 579, 2010.

ASSEMBLÉIA LEGISLATIVA DO ESTADO DE SANTA CATARINA. Lei $n^{\circ} 12.774$, de $1^{\circ}$ de dezembro de 2003: obriga as redes de refeições rápidas de opções restritas, estabelecidas no Estado de Santa Catarina, a informar a seus clientes a quantidade de valor calórico e nutricional contida nas suas refeições . Disponível em: http:// www.alesc.sc.gov.br. Acesso em: 04 abr. 2009.

ASSEMBLÉIA LEGISLATIVA DO ESTADO DE SANTA CATARINA. Lei $n^{\circ} 15.447$, de 17 de janeiro de 2011: dispõe sobre a obrigatoriedade de informar aos consumidores sobre os ingredientes utilizados no preparo dos alimentos fornecidos por restaurantes, bares, lanchonetes, confeitarias, padarias, rotisserias e congêneres que comercializam e entregam em domić́lio alimentos para pronto-consumo, estabelecidos no Estado exercer o direito de escolha alimentar de acordo com as necessidades dos consumidores.

Cabe, portanto, a mobilização da sociedade e, especificamente, dos profissionais de saúde no cumprimento do direito à informação alimentar e nutricional em restaurantes, bem como ao poder público regulamentar e fiscalizar o fornecimento destas informações. Além disso, é importante a conscientização das indústrias de alimentos, funcionários e proprietários de restaurantes quanto à importância da contratação de profissionais para elaborarem estas informações, disponibilizando-as de maneira correta e acessível.

de Santa Catarina, e adota outras providências. Disponível em: http://www.alesc.sc.gov.br. Acesso em: 10 fev 2011.

BENSON, W. Strategies and willingness of rural restaurateurs to promote healthy foods. Can J Public Health, n. 86, p. 181-184, 1995.

BISFELD, B.L; PASTORINO, A.C; CASTRO, A.P.B.M; YONAMINE, G.H; GUSHKEN, K.F; JACOB, C.M.A. Conhecimento da rotulagem de produtos industrializados por familiares de pacientes com alergia a leite de vaca. Rev Paul Pediatr, v.27, n.3, p.296-302, 2009.

BRASIL, Lei 10.674, de 16 de maio 2003: obriga que os produtos alimentícios comercializados informem sobre a presença de glúten, como medida preventiva e de controle da doença celíaca. Diário Oficial da União. Brasília, DF, 19 maio 2003.

BRASIL. Constituição da República Federativa do Brasil. Brasília: Senado Federal, 1988.

BRASIL. Lei no 11.346, de 15 de setembro de 2006: cria o Sistema Nacional de Segurança Alimentar 
e Nutricional - SISAN com vistas em assegurar o direito humano à alimentação adequada e dá outras providências. Diário Oficial da União. Brasília, DF, 18 set. 2006.

BRASIL. Ministério da Saúde. Guia alimentar para a população brasileira: promovendo a alimentação saudável. Brasília: Ministério da Saúde, 2006.

BRASIL. Ministério da Saúde. Política Nacional de Alimentação e Nutrição. 2.ed. Brasília: Ed. Ministério da Saúde, 2003b.

BRASIL. Portaria SVS $n^{\circ}$ 29, de 13 de janeiro de 1998: aprova o Regulamento Técnico referente a Alimentos para Fins Especiais. Diário Oficial da União. Brasília, DF, 30 mar. 1998.

BRASIL. Resolução-RDC n $n^{\circ}$ 60, de 23 de dezembro de 2003: aprova regulamento técnico sobre rotulagem nutricional de alimentos embalados, tornando obrigatória a rotulagem nutricional. Diário Oficial da União. Brasília, DF, 26 dez. 2003a.

BURTON, S.; CREYER, E. H.. What consumers don't know can hurt them: consumer evaluations and disease risk perceptions of restaurant menu items. Journal of Consumer Affairs, v.38, n.1, p.121-146, 2004.

BURTON, S.; CREYER, E. H.; KEES, J.; HUGGINS, K. Attacking the obesity epidemic: the potential health benefits of providing nutrition information in restaurants. American Journal of Public Health, v.96, n.9, p.1669-1675, 2006.

CALIFORNIA CODES. Health and Safety Code section 114087-114094. Disponível em: http://www. leginfo.ca.gov. Acesso em: 15 jul. 2009.

CÂMARA DOS DEPUTADOS. Altera 0 art. $6^{\circ} \mathrm{da}$ Constituição Federal, para introduz̧ir a alimentação como direito social. Disponível em: http://www.camara. gov.br. Acesso em: 08 mar. 2010.

CÂMARA LEGISLATIVA DO DISTRITO FEDERAL. Lei $n^{\circ}$ 2.812, de 30 de outubro de 2001: obriga os restaurantes self-services e estabelecimentos afins a fixarem a quantidade média de calorias das porções dos alimentos. Disponível em: http:// sileg.sga.df.gov.br/sileg/. Acesso em: 04 abr. 2009.
CÂMARA MUNICIPAL DE SOROCABA. Lei $n^{\circ} 7.555$, de 07 de novembro de 2005: dispõe sobre a obrigatoriedade de restaurantes fast foods, bares, lanchonetes, traillers e estabelecimentos similares divulgar informações e tabelas nutricionais sobre os alimentos que vendem e dá outras providências. Disponível em: http://www. camarasorocaba. sp.gov.br. Acesso em: 15 jul 2009.

CÂMARA MUNICIPAL DO RIO DE JANEIRO. Lei $n^{\circ} 3.731$, de $1^{\circ}$ de abril de 2004: dispõe sobre a obrigatoriedade da especificação e divulgação da quantidade de calorias nos cardápios de bares, hotéis, restaurantes, fast-foods e similares. Disponível em: http://www.camara.rj.gov.br. Acesso em: 15 jul 2009.

CARNEIRO, H. Comida e sociedade: uma história da alimentação. v.1. Rio de Janeiro: Campus; 2003.

CLAY, J.M; EMENHEISER, D.A.; BRUCE, A.R. Healthful menu offerings in restaurants: a survey of major U.S. chains. Journal of Foodservice Systems, n.8, p.91-101, 1995.

COMMISSION OF THE EUROPEAN COMMUNITIES. Impact assessment report on nutrition labeling issues SEC(2008) 94.Bruxelas, jan. 2008. Disponível em: http://ec.europa.eu. Acesso em: 10 abr. 2009.

DIN, N.; ZAHARI, M.S.M; SHARIFF, S.M. Nutritional labeling in malaysian full service restaurant menu. Journal of Asian Behavioural studies, v.1, n.3, set. 2011.

EDWARDDWARDS, J. S.; MEISELMAN, H. L. The influence of positive and negative cues on restaurant food choice and food acceptance. International Journal of Contemporary Hospitality Management, v. 17, n.4, p.332-344, 2005.

FISCHLER, C. Food, self and identity. Social Sciences Information, v. 27, n. 2, p. 92-275,1988.

FITZPATRICK, M.P.; CHAPMAN, G.E.; BARR, S.I. Lowerfat menu items in restaurants satisfy customers. JAm Diet Assoc, n.97, p.510514, 1997. 
FOOD AND DRUG ADMINISTRATION. HHS Tackles Obesity. 2004. Disponível em: http:// www.cfsan.fda.gov . Acesso em: 30 jun. 2010.

HWANG, J.; LORENZEN, C. L. Effective nutrition labeling of restaurant menu and pricing of healthy menu. Journal of Foodservice, v.19, n.5, p. 270-276, 2008

JACOBSON, M.F. The hidden cost of eating out. Nutrition Action Health Letter, v.2, p.1-3, 2004.

JOMORI, M.M; PROENÇA, R.P.C.; CALVO, M.C.M. Determinantes da escolha alimentar. Rev. Nutr, v.21, n. 1, p.63-73, jan./fev. 2008.

LANDO, A.M; LABINER-WOLFE, J. Helping consumers make more healthful food choices: consumer views on modifying food labels and providing point-of-purchase nutrition information at quick-service restaurants J Nutr Educ Behav, n.39, p.157-163, 2007.

macronutrient intakes. Public Health Nutrition, v.3, n.2, p. 201-209, 2007.

MAESTRO, V.; SALAY, E. Informações nutricionais e de saúde disponibilizadas aos consumidores por restaurantes comerciais, tipo fast food e full service. Ciênc. Tecnol. Aliment, v.28, supl, p.208-216, dez. 2008.

NESTLE, M. Health Care Reform in Action Calorie Labeling Goes National. N Engl J Med, v.362, p. 2343-2345, jun. 2010.

NEW YORK CITY. Department of health and mental hygiene board of health. Notice of adoption of an amendment (\$81.50) to article 81 of the New York. City Health Code. Disponível em: http://www.nyc. gov. Acesso em: 15 jul. 2009.

POULAIN, J.P. Sociologias da alimentação: os comedores e o espaço social alimentar. Proença,
R.P.C; Rial, C.S; Conte, J, tradutores. Florianópolis: Ed. UFSC; 2004.

PROENÇA, R.P.C; SOUSA, A.A; VEIROS, M.B; HERING, B. Qualidade nutricional e sensorial na produção de refeições. Florianópolis: Ed. UFSC, 2005.

STUBENITSKY, K.; AARON, J. I.; CATT, S. L.; MELA, D. J. The influence of recipe modification and nutritional information on restaurant food acceptance and

TANDON, P.S.; WRIGHT, J.; ZHOU, C.; ROGERS, C. B.; CHRISTAKIS, D.A. Nutrition menu labeling may lead to lower-calorie restaurant meal choices for children. Pediatrics, v. 125, n.2, p. 244-248, fev. 2010.

THOMAS, L. Jr; MILLS, J.E. Consumer knowledge and expectations of restaurant menus and their governing legislation: a qualitative assessment. Journal of Foodservice, v.17, n,1, p.6-22, 2006.

UNITED STATES OF AMERICA. Patient Protection and Affordable Car Act. Section 4205: nutrition labeling of standard meu items at chain restaurants. mar 2010. Disponível em: http://docs. house.gov. Acesso em: 15 jul. 2009.

WOOTAN, M.G.; OSBORN, M. Availability of nutrition information from chain restaurants in the United States. Am J Prev Med, v.3, n.30, p. 266-268, 2006.

WORLD HEALTH ORGANIZATION. Global Strategy on Diet, Physical Activity and Health. Genebra: WHO, 2004a.

WORLD HEALTH ORGANIZATION. Nutrition labels and health claims: the global regulatory environment. Genebra: WHO, 2004b. 
